\title{
Integrated Approach to Text Analysis in Teaching Translation at Linguistic Department
}

\author{
Tatyana Nikitina, and Klara Kasatkina* \\ Togliatti State University, Humanitarian Institute, Togliatti, Russian Federation
}

\begin{abstract}
The article addresses the issue of preliminary translation as part of the translation process. Though the review of scientific discussion in the area shows substantial research of text analysis, its application in teaching practice still remains unclear. Particularly, it concerns the format and effectiveness of different methods. The article describes an integrated model of text analysis, which is based on textological and intercultural approaches and uses different tools for analyzing the text. The model is tested in the practice of teaching translation at the university by the use of questionnaires, a protocol method, and a comparative method. The study of the results proves the efficiency and flexibility of the model in reference to different types of texts. It can be used for different levels of translation training. The application of the integrated text analysis provides a better understanding of the text contents and better equivalency. It also diminishes mistakes in translation. The model of integrated text analysis is a useful tool for educators in linguistic area.
\end{abstract}

\section{Introduction}

A modern economic situation based on cooperation between representatives of different countries creates the need for competent translators to facilitate social, cultural and economic activities. Market demands for translation include speed and quality combined in a reasonable and satisfactory balance [1]. Moreover, there is a unique situation in the modern world, when the rise in migration and international cooperation increases the speed of communication between representatives of different cultures.

Since translation plays a decisive discursive role in organizations that influence the world development, and there is a need to perform the translation in limited time with good quality, scientists and educators search for effective methods to train translators in realistic market situations and equip them with effective tools for fulfilling the task [2]. Scientific discussion about the study of achieving high-quality translation brought to the development of optimal strategies in translation for professionals and students [3]. High-quality translation is mostly achieved by strong editing [4]. Nevertheless, the other components of the translation process (preliminary text analysis or draft translation) are not quite clearly defined in their overall task of achieving high-quality translation.

The aim of the present research is to describe and examine an integrated model of preliminary text analysis for translation purposes, which can be applied in the area of written translation in the education sphere and teach the students to optimize their translation work. The hypothesis of the research is the effectiveness of the integrated text analysis, which can be seen in diminishing the number of translation mistakes causing economy of time and higher translation quality.

\section{Text analysis in theory and practice}

Scientific research in the area of text analysis is quite substantial. Since the start of the text studies, there was the development of text analysis methods for research and other pragmatic purposes.

The hermeneutics approach to text analysis presupposes attentive reading for the complete understanding of all aspects of the text. Philological reading uses the linguo-stylistic method, which leads a researcher and or a translator to discovering all the peculiarities of the text; accordingly, translation and understanding are closely connected. Namely, translation can serve as a way of understanding [5]. Different linguistic aspects of the text, its prosodic, semantic and syntactic organization are thoroughly analyzed to determine the primary or secondary role of linguistic units in creating the text ideology and pragmatics [5]. This method is traditionally applied to literary works, though the general scheme of analysis or some of its components can be applied to other kinds of texts. Such analysis at the lexical level clarifies the semantics of the words, their stylistic and connotative charge, phraseology and cultural reference. The method also helps to reveal morphological and syntactic peculiarities of the text, its rhythmic arrangement. Within this approach, the text is also examined for its stylistic potential. At the metasemiotic level, the philological reader can disclose stylistic devices and expressive

Corresponding author: humanhuman2009@yandex.ru 
means in the text [6]. The complexity of the text semantics brought to the study of the sub-context and implication.

The cognitive analysis examines the text in the area of concepts, ideas and cultural reference, which often demands looking for additional information [7]. The examination of the concepts and cultural background in a linguo-cultural method directs text analysis outside the text to create a complete picture of it $[8,9]$.

The same direction can be seen in the textocentric analysis, where a text is understood as an element of text activity created by the text, its reader, its writer and the surrounding reality and, accordingly, such analysis must refer to these participants [10]. Besides, the interaction between the author, the audience and the text can be examined by rhetorical analysis.

Pragmatics of the text is disclosed in discourse analysis, where the text is viewed as a tool of communication between the reader and the writer [11, 12]. Discourse methods are effective in discovering all logical, experiential, interpersonal and textual meanings of the text. Nevertheless, discourse analysis is very labor-intensive. Thus, the research and development of methods of text analysis brought linguistics to the study of the text as a unit of communication.

The changes in modern text usage and forms brought forth new methods of text analysis. Thus, due to development of computer technologies and various applications modern linguistics is also equipped with computer-assisted methods of research. Text analysis development is not an exception [13-15]. The Internet Age promoted greater speed of translation, which can be achieved by using different machine-assisted tools of translation and analysis [1]. Thus, for example, there are computer tools for analyzing the morphological level of the text, and disclosing categorical divergence in parts of speech distribution typical for a particular Source Language and a Target Language [14]. The use of translation programs makes it possible to make the analysis of structure, meaning, and thematic divergence. Another example could be the use of content analysis for modern media and Internet hypertext [16]. Content analysis allows processing vast volumes of the texts through examining and decoding themes, structures, features, links and exchanges taking into account different semiotic systems.

Though the above-mentioned methods provide an accurate understanding of the text, they are, nevertheless, not directed at translation, since they were designed for other purposes. The theory of text analysis for translation does not deny the existing methods but develops and modifies them for translation purposes. Thus, M. Szczyrbak explains that teaching translation should rely on both market needs and the students' needs [2]. Accordingly, education becomes the main objective rather than training: it is necessary to create a framework for professional development rather than impose a set of practical skills. In this respect, multifunctional translation models can help to achieve the goals of translator's education.

The need for such models is clearly explained by one of the founders of translation-oriented text analysis Chr.
Nord, who writes about the need to create a general and at the same time flexible model of text analysis, which can be adjusted to a particular translation situation [3]. Translation-oriented text analysis should also be applicable to professional experience and didactic situations of translation classes [17].

Nord and other scholars underline the importance of taking cultural aspect of translation into account [3, 1820]. Chr. Nord explains the mechanism of intercultural text transfer, which happens during translation through the notion of the initiator, who transfers the text to a different culture, while the text is seen as means of communicative interaction [3]. As for the translator's role, it can be explained through the concept of intermediation, applied to the development of media and its effect on sensory perception [21]. Media transforms the original text and conditions its perception, which is similar to the translator's mediating role. It is important to mention that the changes of media context cause the changes in perception, which can be also observed in the translation context, where different translations can condition different perception. The idea of a translator as a mediator in intercultural communication explains an important role of the cultural element in text analysis [9, 19]. S.E. Pommer introduces the term "cultural immersion", which presupposes that translation in certain areas, for example, in the legal area is greatly dependent on unique national contexts [9]. Thus, the translator becomes a provider of communication between cultures. This logically leads to exploring the cultural background of the Source Text (ST) to provide an adequate Target Text (TT). The basic principles of translation-oriented text analysis are, first, the interconnection of the intratextual and the extratextual and, second, the function of the ST in culture. Consequently, one of the main aims of text analysis is to identify and isolate the elements, which must be preserved and adopted in translation.

As for the mechanism and components of the translation-oriented methods, there are different models presented in scientific research. For example, Nord applies text analysis to the whole of the translation process, since even in the process of creating a translation there is an analytical operation of transcoding [3]. Other researchers use the term "text analysis" only in application to preliminary analysis [11, 18-20].

In spite of the fact that the nature of translationoriented analysis is understood differently, there is agreement about its two basic goals and two stages: the first one aims at general comprehension, the second one is focusing on relevant elements that need reproduction according to the skopos theory $[3,11,17,18-20]$. Citing H. Vermeer, Chr. Nord describes the matrix of text analysis as the study of attitude, status, role, strategy, behavior and activity, which is applicable to the particular communicative situation and to the situation of translation [3]. J. House notes that the information presented through these discursive categories is modified and displayed differently [22]. The study of all aspects of the text is very important for understanding its pragmatic side, its intentions. Thus, for example, the study of attitude in investigation context revealed its great role in 
facilitating communication between the investigator and the suspect [23]. Though such empathy is not directly articulated, but hidden in the context, it has to be taken into account when considering the text translation. Attitudes can be also examined in terms of multimodality, which explains how the text is defined by its message [18]. Modern pragmatic theories of translation expand the skopos theory by $\mathrm{H}$. Vermeer explaining such scenarios of translation, in which the translator changes the elements of the ST.

The application of different communication and translation theories to text analysis was very successful in particular areas of teaching translation $[9,11,12,17]$. The study of the discursive-communicative approach to translation in a didactic context shows that the aims of text analysis for professional translation and for education are different. Analyzing the text in class demonstrates how applying components of different analysis methods can be useful in translation activity, and in what way the student can combine these methods to fulfill different translation tasks. In the didactic context, there is no strict limit of time for translation tasks and student translators do not always reach the final result. To sum up, educating purposes are primary in using text analysis in class, while the idea of integrating different methods serves the aim of expanding students' knowledge of different approaches.

Relying on the above-mentioned tools of text analysis and approaches to studying the text and translation process, the present article offers an integrated model of translation-oriented text analysis for education context. The model is based on pragmatic approaches to text and translation, as well as the theory of intercultural communication. The tools of such preliminary analysis are borrowed from the skopos theory, the functional stylistics and the text theory $[1,3$, $6,8]$. In these approaches and theories, the text is seen as a component of discursive communication, where the translator is a mediator conveying the text semantics and functional potential. The model corresponds to the notion of process-driven pedagogy [24]. In this respect, the model is seen as means of developing critical views and performative skills through encouraging initiative, discussion and self-development.

The integrated model of text analysis is applicable only to preliminary analysis which precedes the process of translation. Nevertheless, its two aims are directed at both the preliminary reading and translation: the first one is to comprehend the text in the complexity of its information content, while the second one is to promote translation decisions. Accordingly, text analysis is divided into two stages: the stage of comprehensive reading and the stage of prognosis. These two stages are based on the strategies of optimizing, analyzing and adapting.

Using the optimizing strategy the student learns to achieve the optimal balance of time and quality taking into account the situation of translation. The strategy is integrating approaches used in pragmatics, computation and time-management. For example, some translation does not demand the highest quality if it is needed for surface summary or review. In other cases, there is a need for thorough analysis and high quality, since translation services high-responsibility activities in manufacture or financing. The quality of translation can be connected with time. Thus, for example, preliminary translation of project documents for assessing the projector's potential can be characterized as fast, but the volume of precision information is not very high, thus, the translator may focus not on high accuracy, but on positive modality. When the project is already in work, complete translation of the documents is needed. It is time-consuming, and, accordingly, it needs higher accuracy and quality. The recipients can also define the quality of translation. Texts for professionals are scrutinized for quality; texts for public use are also examined for accuracy. The students need to plan the optimal balance of time and quality at the stage of text analysis.

Optimizing strategy can also employ different tools of translation. Students need to learn that, for example, the use of machine translation greatly decreases the time for translation, but the quality can be lower. Thus, during comprehensive reading, the translator must determine the proportion of machine and human translation. Some texts with simple informative structure can be easily translated with the help of computer programs with the minimum editing by the translator, other texts can be translated when they are adopted for machine translation. Texts with the complicated informative structure or a great proportion of idioms and set expressions are preferably translated by people. The choice of the wrong balance can increase the time of translation and damage the quality.

In the integrated model, the optimizing strategy is employed during comprehensive reading and also the prognosis part. Students learn to understand that predicting translation decisions for certain linguistic units and defining similar decisions for groups of similar units can greatly optimize the translator's work. To illustrate this, the choice of similar translation decisions for dealing with abbreviations (decoding or finding equivalents) can lead to uniformity of the translated text and optimizes the work on translation.

The students of translation should also employ the strategy of systematizing. It is based on integrating different linguistic disciplines, communication and computation. This strategy transforms the text into the system of interrelated informative elements. These elements can be intratextual and extratextual [3]. They can be discovered in the narrow and wide contexts [25]. Presenting the text as a system of verbal and non-verbal means in their interrelation can help the students to understand how to organize their translation work with information, which is split into two forms of presentation. Inscriptions to illustrations, advertisement slogans, scheme inscriptions may have different degrees of connectedness with the graphics and illustrations. The study of the text as a system of compositional elements teaches students how information is displayed in the ST: argumentation, narration, description etc. It can also be important for spacial arrangement and format of the text, if this is included in the translation task. 
Students who translate should understand that systematizing at the linguistic level is very important since comprehension also involves understanding the main linguistic peculiarities of the text. Students learn to see that phonetic, grammatical, lexical and stylistic levels of text analysis reveal those translation units, which can cause difficulties in translation. They should learn to find the best approaches to translating them. Such units are, for example, verbal constructions or terminology. It is advisable to encourage students to make a glossary of translation equivalents for these units and a chart of translation decisions for similar units. Students should discuss the ways of translating lacunas and linguistic experiments like word-play. Students should also learn to see the functional priority of these units. For example, the rhythmic arrangement can be a priority for translating poetry, but it is irrelevant for a business document. In the same way, an instruction does not contain stylistic devices, while a magazine column uses them as important means for making speech more expressive.

Within this strategy, students learn to use the computer-assisted tools. For example, the word count can help to predict the volume of translation, especially if there are some limiting parameters for the volume. This can happen in case the text is placed in space frames on the designed page. Since Russian and English languages have different parameters for the word volume, during text analysis the translator must predict what components of the text could be subject to compression, leaving precision information intact. Comprehensive reading could also be assisted by sentimental analysis, which reveals the emotional frame of the text. Accordingly, students learn to define the character of the ST as sentimental or neutral.

Finally, presenting the text as a pragmatic system of goals, intentions and modalities develops the skill of defining the text role in communication process. For example, discovering the intentions of a scientific text as informing, persuading, arguing or explaining, as well as their balance and priority, can help to reconstruct the pragmatic frame of the ST in translation. A pragmatic system also includes the examination of various textual categories: expressiveness, intertext, integrity, coherence and cohesion.

The adoptive strategy is connected with the need to adopt the TT to a certain recipient or discursive situation. This strategy integrates the approaches of pragmatics, cultural and social studies. To employ the adoptive strategy, students need to understand the admissibility of changes in the text, which is achieved through comprehensive reading. It includes the material of the text or any other texts defining the information in the ST. These could be encyclopedic dictionaries, mass media articles, scientific papers and other necessary materials. Thus, the translation of the scientific article could be assisted by reading about a scientific discussion in a certain area. The translation of a literary text must be assisted by bibliographical, cultural, historical and other references. The volume of additional reading is variable depending on the stylistic type of the text.
Students should also learn to take into account the hypertext potential of the text. Thus, court papers are part of an investigation case; they are understood and modified only within the case context. Parodies can be translated and adopted only on the basis of the original.

Another factor determining the need for additional reading is cultural reference. Specific national frame, description of customs and traditions, the appearance of lacunas demand additional reading. The translator must define the role of the culturally relevant information in the ST and predict its impact on the reader of the TT.

The use of the three strategies helps the teacher of translation to lay out a way for achieving translation goals. The use of the model increases the range of tools for analyzing the text and develops such professional qualities as responsibility, independence and industry. The model is flexible: it can be applied to different didactic contexts in the complete form or as a collection of components. The hypothesis of the present research about the effectiveness of the model is proved through the examination of students' translation practice.

\section{Method}

The study of the integrated model is performed through the use of qualitative and quantitative methods in two stages. The first stage included the questionnaire with the aim of examining the need for integrating different approaches and the application of integrated analysis to students' practice of translation. During the second stage, practical work of students was examined to discover the interconnection of the translation quality and the integrated method of translation-oriented text analysis.

Since translation is a complicated and many-sided process, the issue of proper translation analysis is a disputable question [1]. Though there is a universal agreement that, in spite of the deadlines, the quality level in modern translation must be high, the systems of assessment in modern translation theories are numerous [1]. They offer different pragmatically relevant models, which can make a fast general assessment or a specific detailed one to a lot of different parameters taking into account the text type, extraneous factors, errors, accuracy and quality.

In creating the educational context for student's translation, the present research relied upon such components as the communicative situation or "field", participants, mode of communication, genre disclosure and comparison of ST and TT [1]. The examination of translation mistakes relies upon the principles of $\mathrm{P}$. Kussmaul and examination of mistakes in reference to their negative effect on the overall quality of the translation [26]. The basis of mistake assessment is the classification of V.N. Komissarov, where the mistakes are differentiated into translational ones (damaging or distorting the information) and linguistic ones (diminishing quality through linguistic or stylistic parameters) [25]. This negative effect can be different depending on the volume of the text affected by the 
error. The research results and the integrated model were discussed with the students during classes of translation.

\section{The field study of the integrated analysis in teaching translation}

In order to understand the needs of future translators, a questionnaire was conducted on the basis of a sample group of students of linguistics in order to clarify the role of integration in translation practice and the areas that can suggest such integration. A sample volunteering group of 48 day-time students of the linguistic departments answered the questions in terms of anonymity to ensure that their answers are not influenced by their teachers and they can report their translation experience accurately.

The questionnaire aimed at discovering the need for integrating the methods and information from different disciplines and theories. The questions about the use of different methods of analysis showed that the respondents knew about at least two of the offered text analysis methods discussed in the present article, but their practical application was limited mostly by the preferable use of linguo-stylistic and linguo-cultural methods, which 45 of the respondents combined during preliminary analysis (93, $7 \%$ ). Linguo-stylistic method was acknowledged to be the most optimal one by 78 students $(95 \%)$.

Answering the question whether any additional information about the text was needed during translation analysis, all the 48 respondents $(100 \%)$ answered that they needed additional knowledge. Choosing from the options offered they chose areas of science they needed to rely upon in preliminary text analysis.

Table 1. Additional areas of study needed during preliminary text analysis.

\begin{tabular}{|c|c|}
\hline Area & $\begin{array}{c}\text { No of } \\
\text { respondents/ } \\
\text { percentage }\end{array}$ \\
\hline Linguistics & $38 / 79.1$ \\
\hline Cultural Studies & $41 / 85.4$ \\
\hline $\begin{array}{c}\text { Technical } \\
\text { Sciences }\end{array}$ & $12 / 25$ \\
\hline Communication & $16 / 33.3$ \\
\hline Social Studies & $20 / 41.6$ \\
\hline
\end{tabular}

The questions about aspects of text analysis offered students to point what aspect or aspects of the text they, first of all, focused on. The options for choice included lexical, grammatical and phraseological difficulties, the structure of the text, subcontext, cultural background and pragmatic adaptation. The questions about the focus of text analysis on different aspects of the text and its environment showed that 38 students $(79,1 \%)$ pay attention to three aspects of the text during text analysis. 23 students $(47,9 \%)$ chose grammatical difficulties, 18 students $(37,5 \%)$ chose lexical difficulties and 15 students $(31,2 \%)$ chose phraseological difficulties. Thus, during comprehensive reading before translating the text $47,9 \%$ of students are aware of linguistic features of the text causing possible problems in translation. As for the structure of the text, it is examined only by 5 students $(10,4 \%)$. During comprehensive reading 24 students $(50 \%)$ also pay attention to the cultural background. Only 3 and 5 students accordingly focus on pragmatic adaptation and subcontext (6\% and 10,4\%).

The second stage of the experiment included the comparison of translations made on the basis of preliminary reading and translations made with the use of integrated text analysis by two groups of students (27 and 21 correspondingly). The translations were examined for the average number of mistakes per 1000 characters of the text. The mistakes were registered in protocols for examination. The study of protocols with translation mistakes showed that the average mistake count in translation performed without integrated analysis was 7,2 mistakes per 1000 characters of the translated text. The use of integrated analysis diminished the average number of mistakes to $2-6$ mistakes.

The study of the mistake types showed, first of all, the prevalence of mistakes related to the cultural background of the text and understanding the implied meaning, that is, the mistakes of the subcontext or extratextual mistakes. Thus, for example, in the translation of the text about Russian history by Isaiah Berlin the phrase "young révoltés" is translated as "молодые повстанцы", while the analysis of extratextual information about the historical period described in the text can lead to a correct translation "молодые революционеры". The phrase "Russian ladies" translated as "российские дамы" showed that the student translator did not differentiate the words "российский" and "русский", which is not only a lexical mistake but also a cultural one. The translation of the phrase "continent generation" as "континентальное поколение" in the first group was changed into “молодежь Европы” after integrated analysis.

Superficial reading of the text about Russian culture and traditions in the first group imposed false acculturation of the TT. To illustrate, the phrase "psychological and aesthetic patterns" was translated with the word "узоры", since it seemed to be connected with the traditional Russian handicrafts, though the study of the cultural background could suggest the correct translation "предпочтения", since the phrase described the influence of Western culture on Russian thinkers.

Besides mistakes of cultural character, the integrated analysis diminished the number of mistakes connected with linguistic and stylistic norms. For example, incorrect translation of "cut-and-dried" as "высушенные" instead of "шаблонные" or the translation of "young and fertile" as "молоды и детородные" violating grammatical and stylistic norms.

The translation assessment, possible equivalents and variants of improvement were discussed by students. The 
classes where the students translated the texts and analyzed translations were organized on the basis of the skopos theory with the tasks of preserving all the norms typical for a particular genre of the text [27]. Localizing the text in translation becomes more realistic since the teachers created a situation of translation similar to the professional one, where the role of the translator is determined by the general or micro-tasks. The students were also offered the opportunity to analyze mistakes in mini-groups and individually, discuss them and correct. It followed the approach of P. Kussmaul, who suggested the focus on the translation problem [26]. For example, in the translation of advertisements and their further analysis with the help of the protocols students distinguished the problem of translating slogans.

In their discussion, the students came to the conclusion that resorting to extratextual information improves the quality of the text. Students correctly identified the mistakes connected with the distortion of meaning and with the wrong translation of culturally loaded units. Thus, the translation of the slogan "Without the right solution you won't get far" by Infonet company was accompanied by the picture of a policeman measuring speed. The students who did not employ the integrated method made a translation with the focus on the vehicle, while the opposite group made a correct translation on the basis of information about money transfer by the Infonet company. In the translation of the slogan by Country Floors, which contained the idiom "still waters run deep" the first group used an equivalent for the idiom, while the second one found information about the company and translated the slogan with the focus on the floor quality.

Students also discussed the results of computerassisted sentiment analysis with the help of Python NLTK online resource, which showed 0,7 polarity of the text against 0,3 neutrality, positive sentiment polarity being 0,9 . The analysis of the translation after comprehensive reading showed greater neutrality, which the students of the first group explained by perceiving the text as scientific and unemotional. The use of sentiment analysis during integrated analysis allowed correcting the results to bring them closer to the characteristics of the ST.

The application of the model made translation classes dynamic and creative. Discussion of translated problems and mistakes helped students to see the microstrategies that were miscalculated during text analysis.

\section{Discussion}

The study of the questionnaire showed that text analysis, which students used preliminary in translation, demonstrates the need for other linguistic and momlinguistic areas needed for complete understanding. These areas mostly include linguistics, cultural studies and social studies. They are connected with cultural difficulties and the social context, where the text, the author, the translator and the recipient condition the parameters of the translation process.
The study of text analysis methods applied in translation experience in the questionnaire demonstrated the limited use of analysis tools. The students resort mostly to linguo-stylistic method and consider it optimal for translation. Nevertheless, understanding the need to apply different methods grows with the advancement of translation experience. This is, probably, connected with the more complicated nature of the texts that students deal with at the advanced level of professional training.

Examination of the text analysis technique and focus, as it is represented in the questionnaire, showed that understanding the need for deeper integrated analysis is stronger with the advancement of professional training and experience.

The study of the mistakes made in translation demonstrated their dependence on the application of the integrated text analysis model. The application of the model brought to diminishing the number of mistakes in the text in general. It also diminished the translation mistakes, conditioned by misunderstanding the cultural information hidden in the subcontext.

The positive by-side effect of applying integration analysis is the growth of professional awareness and interest. It is provided by discussion and creative activities that engage students in realistically reconstructed professional situations. Thus, the integrated model can be considered effective, leading to better quality of students' translation.

\section{Limitations}

Considering the limitations of the study, it is necessary to note that the course of translation is universal for many educational institutions; nevertheless, the course design, as well as the theoretical framework for didactic component, may be different. Thus, some particular results of the study, which are relevant for the sample group of students, may be inapplicable to students at other educational institutions. Limitations are also connected with the texts used for the study. Different temporal and functional parameters for the text choice can probably make the results of the study more variable in terms of the flexible components of the text analysis model, which can be tested in further research of translation-oriented text analysis.

\section{Conclusion}

The study of scientific discussion and field experiments showed that integrating theories and methods from different scientific areas could help students in translation to develop their professional skills and achieve high-quality translation through a flexible model of preliminary text analysis. The survey of scientific works in the sphere of text analysis showed that text analysis for translation purposes is based on linguistic, cognitive, pragmatic and content methods, assisted by computer tools. Nevertheless, analysis for translation purposes is thoroughly specific and relies upon the discursive and intercultural frame of communication, where translator plays an important mediatory role. Such 
analysis is most effective when it integrates the tools and methods from different disciplines: cross-cultural studies and communication.

The didactic model of integrated text analysis is based on the discursive and cultural approaches and includes the tools of analysis from the areas of stylistics, social studies, cultural studies and computation. The proposed model of integrated text analysis aims at developing professional competence of students. It was supported by the study of integration needs of translation students and by testing the translations performed with the use of integrated text analysis. Both questionnaire and assessment of translations showed the need for application of this model and the effectiveness if it.

Further study of the integrated model of text analysis can be applied to different groups of translation students giving more variables in levels of training and professional areas. The study of different kinds of text can lead to defining the specification of the model as it is adjusted to a particular translation context.

\section{References}

1. J. Drugan, Quality in professional translation: assessment and improvement (Bloomsbury, London, NY, 2013).

2. M. Szczyrbak, Reflections on teaching business and legal translation to Polish undergraduate students, SKASE J. Transl. Interpret. 3 (1), 65-81 (2008)

3. Chr. Nord, Text analysis in translation: Theory, methodology, and didactic application of a model for translation-oriented text analysis (Amsterdam, NY, Rodopi, 2005).

4. T.G. Nikitina, The tasks of forming skills of translation-oriented text analysis and editing in professional training of translators, Concept 2, 5561 (2018)

5. V.Ya. Zadornova, LATEUM-2015. Research and Practice in Multidisciplinary Discourse. Conference proceedings (Moscow, 2015)

6. V.Ya. Zadornova, Perception and interpretation of the literary text (Moscow, Vysshaya shkola, 1984)

7. K. Senkbeil, N. Hoppe, Lang. Lit. 25 (1), 3-17 (2016)

8. V.I. Karasik, Language matrix of culture (Moscow, Gnozis Publ., 2013)

9. S.E. Pommer, SKASE J. Transl. Interpret. 3 (1), 1722 (2008)

10. J. Hansen, Transl. Interpret. Stu. 11 (1), 100-117 (2016)

11. T.A. Volkova, Polit. Lingvistika, 3, 17-24 (2011)

12. J. Munday, M. Zhang, Discourse analysis in translation studies (Philadelphia, John Benjamins Publishing Company, 2017)

13. G. Rockwell, Lit. Linguist. Comput. 18 (2), 209-219 (2003)

14. N. Habash, B. Dorr, Ch. Monz, Mach. Translat. 23, 23-63 (2009)
15. Y.R. Tausczik, J. W. Pennebaker, JLS 29 (1), 24-54 (2009)

16. S.C. Herring, Content Analysis for New Media: Rethinking the Paradigm, New research for new media: innovative research methodologies symposium, (USA, 47-66, 2004)

17. M. Morón, E. Calvo, J. Spec. Translat., 29, 126-148 (2018)

18. S. Dicerto, Multimodal pragmatics and translation: a new model for source text analysis (Palgrave Macmillan, Springer, 2017).

19. K.A. Kasatkina, Vector Nauki TGU, Pedagog. Psychol., 3 (26), 27-31 (2016)

20. M.V. Ryabova, Philological science. Theory and practice 1 (3), 170-174 (2009)

21. L. Bertens, Third Text, 30, 90-99 (2016)

22. J. House, Translation quality assessment: a model revisited (Tubingen, Gunter Narr Verlag, 1997)

23. C.J. Dandoa, G. E. Oxburghb, Eur. J. Psychol. Appl. to Leg. Context, 8, 27-33 (2016)

24. S. Kadiu, Curr. Trends Transl. Teach. Learn., 4, 4877 (2017)

25. V.N. Komissarov, Modern translation theory (Moscow, EST Publishing, 2001)

26. P. Kussmaul, Training the translator (Amsterdam, John Benjamins, 1995).

27. H. J. Vermeer, A scopus theory of translation (some arguments for and against) (Heidelberg, Text von Text Verlag, 1996). 